\title{
A coordinative saturated vanadium containing Metal Organic Framework that shows a remarkable catalytic activity
}

\author{
Karen Leus, ${ }^{\mathrm{a}}$ Ilke Muylaert, ${ }^{\mathrm{a}}$ Veronique Van Speybroeck, ${ }^{\mathrm{b}}$ Guy B. Marin, ${ }^{\mathrm{c}}$ \\ and Pascal Van Der Voort, ${ }^{a}$ \\ ${ }^{a}$ Center for Ordered Materials, Organometallics and Catalysis (COMOC), Department \\ of Inorganic and Physical Chemistry, Ghent University, Krijgslaan 281 (S3), 9000 \\ Ghent, Belgium. \\ ${ }^{b}$ Center for Molecular Modeling, Ghent University, Technologiepark 903, 9052 \\ Zwijnaarde, Belgium. \\ ${ }^{c}$ Laboratory for Chemical Technology, Ghent University, Krijgslaan 281 (S5), 9000 \\ Ghent, Belgium.
}

\begin{abstract}
A completely saturated Metal Organic Framework, MIL-47 was synthesized and tested for its catalytic performance in the oxidation of cyclohexene with tert-butyl hydroperoxide as oxidant. The catalyst was compared to several reference catalysts: namely VAPO-5, supported $\mathrm{VO}_{\mathrm{x}} / \mathrm{SiO}_{2}$ and the homogeneous catalyst $\mathrm{VO}(\mathrm{acac})_{2}$. MIL47 shows a remarkable catalytic activity and preserves its crystalline structure and surface area after a catalytic run. Furthermore MIL-47 exhibits a very high activity in successive runs.
\end{abstract}

Keywords: Metal Organic Frameworks, vanadium, oxidation, liquid phase

\section{Introduction}

Metal Organic Frameworks (MOFs) are crystalline porous solids composed of a threedimensional (3D) network of metal ions held in place by multidentate organic molecules $[1,2]$. In recent years, MOFs have received considerable attention as potentially valuable gas storage and catalyst materials [3-7]. MOFs possess several attractive features: a high micropore volume, crystallinity and a high metal content offering potentially valuable active sites.

So far, only a few catalytic applications of Metal Organic Frameworks have been reported. Some of their potential applications were outlined recently in two excellent reviews [8,9]. All these reports deal with Metal Organic Frameworks that have unsaturated sites. However, to obtain insight into the real nature of the active sites, it is of a paramount importance to study saturated Metal Organic Frameworks.

Therefore, a completely saturated, vanadium containing MOF was synthesized, namely MIL-47. This MOF is a porous terephthalate built from infinite chains of $\mathrm{V}^{4+} \mathrm{O}_{6}$ octahedra, held together by dicarboxylate groups of the terephthalate linkers and has a three-dimensional orthorhombic structure [10].

In the present work, we have tested MIL-47 for its catalytic performance in the oxidation of cyclohexene. Amongst the various oxidation products of cyclohexene, cyclohexane epoxide is a highly reactive and selective organic intermediate which is widely used in the synthesis of enantioselective drugs, epoxy paints and rubber 
promoters [11]. Furthermore the catalytic activity of MIL-47 is compared to VAPO-5, $\mathrm{VO}_{\mathrm{x}} / \mathrm{SiO}_{2}$ and the homogeneous catalyst $\mathrm{VO}(\mathrm{acac})_{2}$.

\section{Experimental section}

The hydrothermal synthesis of MIL-47 is based on a literature procedure [10]. A mixture of $\mathrm{VCl}_{3}$, terephthalic acid and $\mathrm{H}_{2} \mathrm{O}$ (molar ratio 1/0.25/100) is brought into a Teflon lined steel autoclave, which is heated at $473 \mathrm{~K}$ for 4 days. In a next step, MIL47 as is brought at $573 \mathrm{~K}$ for $22 \mathrm{~h}$ and 30 min to remove the excess of terephthalic acid in the pores. VAPO-5 is synthesized as described previously: a solution of oxovanadium (IV) sulphate-hydrate and a solution of $\mathrm{H}_{3} \mathrm{PO}_{4}$ are mixed together. While stirring, pseudo boehmite (from Sasol) and triethylamine are added. In a further step, the gel is brought into an autoclave and placed in an oven at $443 \mathrm{~K}$ for 2 days. By centrifugation, the solid is recovered. Furthermore the catalyst is dried and calcinated under a $\mathrm{O}_{2}$-flow [12]. For the synthesis of $\mathrm{VO}_{\mathrm{x}} / \mathrm{SiO}_{2}$, Kieselgel 60 is stirred in a $\mathrm{NH}_{4} \mathrm{VO}_{3}$-solution at $338 \mathrm{~K}$ for $2 \mathrm{~h}$. Afterwards, the solid is filtered and dried during $2 \mathrm{~h}$ at $373 \mathrm{~K}$, followed by a calcination at $823 \mathrm{~K}$ during $5 \mathrm{~h}$.

After a catalytic run, the MIL-47 is regenerated by a treatment in a tubular furnace under a $\mathrm{N}_{2}$-flow at $523 \mathrm{~K}$. This is necessary to remove the organic compounds in the pores.

\section{Results and discussion}

The oxidation of cyclohexene was carried out in a three neck flask under an inert atmosphere. To a solution of cyclohexene (0.05 mol), tert-butyl hydroperoxide $(0,14$ mol) and 1,2,4-trichlorobenzene (0.05 mol) (used as internal standard) in chloroform $(0.38 \mathrm{~mol}) 0,1 \mathrm{~g}$ of the catalyst was added. The reaction mixture was stirred at $50^{\circ} \mathrm{C}$. All the samples were analyzed with a Trace GC Ultra (Finnigan), fitted with an capillary column $(10 \mathrm{~m}, 0,1 \mathrm{~mm}, 0,4 \mu \mathrm{m})$ and an FID detector. Blanc reactions were performed without catalyst.

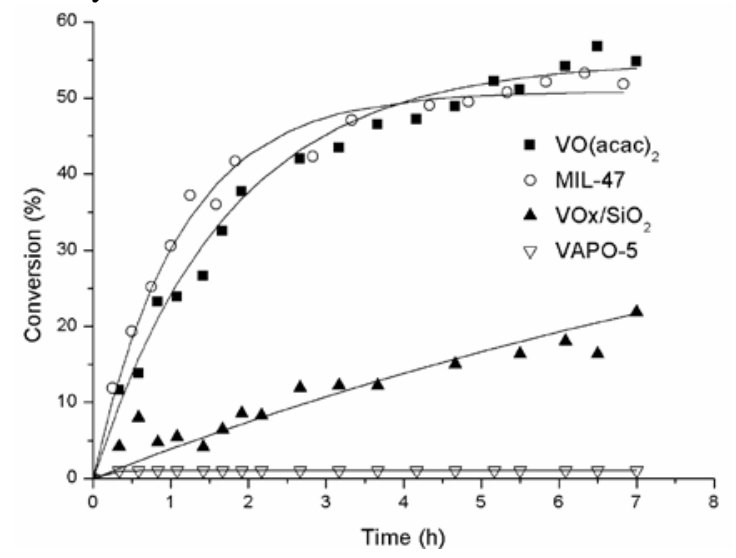

Fig. 1 Conversion curve of cyclohexene for ( $\bullet$ ) unsupported VO(acac) ${ }_{2},(\circ)$ MIL-47, ( $\Delta$ ) $\mathrm{VO}_{\mathrm{x}} / \mathrm{SiO}_{2}$ and $(\boldsymbol{\nabla}) \mathrm{VAPO}-5$.

In Figure 1, the conversion curve of cyclohexene is presented in comparison with the three reference catalysts. As can be seen in Figure 1, VAPO-5 is catalytic inactive for the oxidation of cyclohexene, whereas the three other catalysts: MIL-47, the supported $\mathrm{VO}_{\mathrm{x}} / \mathrm{SiO}_{2}$ and the homogeneous $\mathrm{VO}(\mathrm{acac})_{2}$ exhibit a very high catalytic activity. 
The turn over number (TON) of MIL-47 is calculated, based on the amount of cyclohexene that is converted. The TON of MIL-47 was approximately 108 after eight hours of reaction. Thermal Gravimetric Analysis experiments (TGA) were performed on MIL-47 before and after a catalytic run to quantify the amount of leached vanadium. In comparison with the supported vanadium oxide catalyst, only a small amount of vanadium is leached. The leaching was less than $20 \%$ in the first run with MIL-47, whereas the $\mathrm{VO}_{\mathrm{x}} / \mathrm{SiO}_{2}$ showed a leaching of more then $40 \%$. Furthermore, the catalyst was recovered after a first catalytic run. The X-ray diffraction patterns of MIL-47 before and after regeneration are shown in Figure 2.

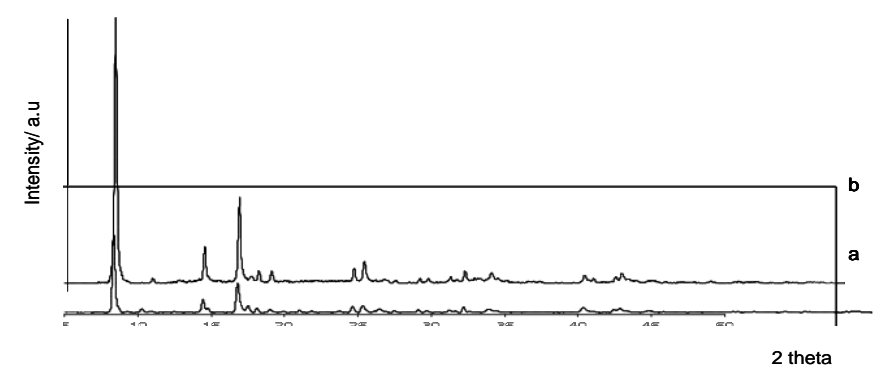

Fig. 2 XRD patterns of MIL-47 (a) before and (b) after regeneration.

MIL-47 preserves its crystalline structure after regeneration, as can be seen from Figure 2. Moreover, the nitrogen adsorption experiments of MIL-47 before and after regeneration are presented in Figure 3. Note that the MIL-47 shows no loss at all of surface area and pore volume after regeneration.

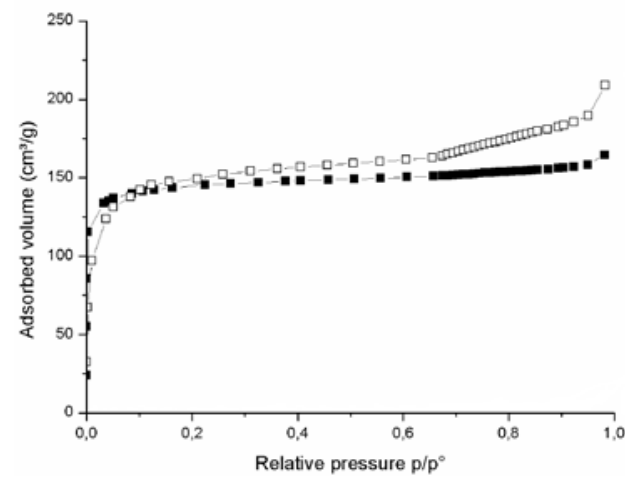

Fig. 3 Nitrogen adsorption isotherms of MIL-47 ( $\square$ ) before and ( $\square$ ) after regeneration.

To evaluate the regeneration capacity of this novel catalyst, MIL-47 was tested for a second catalytic run and compared to the vanadium oxide catalyst. The conversion of cyclohexene for MIL-47 and the $\mathrm{VO}_{\mathrm{X}} / \mathrm{SiO}_{2}$ catalyst in the first and second run is shown in Figure 4. 


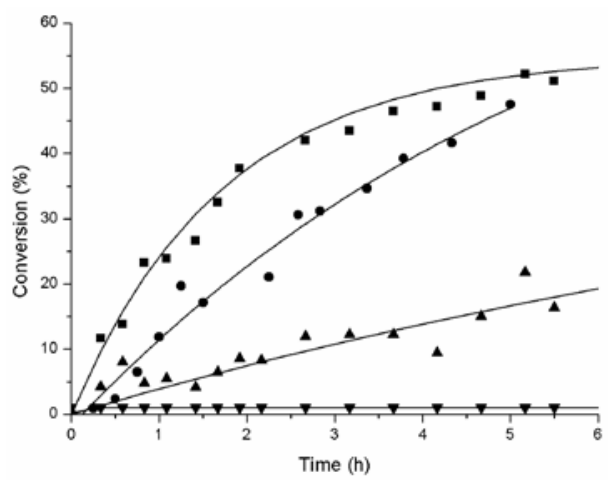

Fig. 4 Conversion of cyclohexene for MIL-47 in its ( $(\mathbf{)})$ first run, $(\bullet)$ second run and $\mathrm{VO}_{\mathrm{x}} / \mathrm{SiO}_{2}$ $(\boldsymbol{\Delta})$ first and $(\boldsymbol{\nabla})$ second run.

MIL-47 still shows a high conversion of cylohexene, whereas the supported $\mathrm{VO}_{\mathrm{x}} / \mathrm{SiO}_{2}$ shows no activity at all in its second run due to leaching of the vanadium centers. This observation indicates that MIL-47 acts as a truly heterogeneous catalyst.

In conclusion, the saturated Metal Organic Framework, MIL-47, is investigated for its catalytic activity for the oxidation of cyclohexene and compared to three reference catalysts. MIL-47, containing saturated vanadium centres, shows a high catalytic conversion. X-ray diffraction measurements and nitrogen adsorption experiments prove the stability of this new catalyst under oxidation reactions. Furthermore MIL-47 exhibits a very high catalytic activity in successive runs.

\section{References}

1 M. Eddaoudi et al, 2001, Modular chemistry: Secondary building units as a basis for the design of highly porous and robust metal organic carboxylate frameworks, Acc. Chem. Res., 34, 4, 319.

2 S.L. James, 2003, Metal Organic Frameworks, Chem. Soc. Rev., 32, 276.

3 C. Janiak, 2003, Engineering coordination polymers towards applications, Dalton Trans., 14, 2781.

4 S. Kitagawa et al, 2004, Functional porous coordination polymers,Angew.Chem. Int.Ed., 43, 18, 2334.

5 Y.M.A. Yamada, et al, 2006, Novel 3D coordination palladium-network complex: a recycable catalyst for Suzuki-Miyaura reaction, Org.Lett, 8, 19, 4259.

6 S.H. Cho et al, 2006, A metal organic framework material that functions as an enantioselective catalyst for olefin epoxidation, Chem. Commun., 24, 2563.

7 B. Gomez-Lor et al, 2005, Novel 2D and 3D indium metal organic frameworks : Topology and catalytic properties, Chem. Mater.,17, 10, 2568.

8 A.U. Czaja et al, 2009, Industrial applications of metal organic frameworks, Chemical Society Reviews,38, 1284.

9 U. Mueller et al, 2006, Metal organic frameworks- prospective industrial applications, J. of Mat. Chem., 16,7,626.

10 K. Barthelet et al, 2002, A Breating Hybrid Organic-Inorganic Solid with Very Large Pores and High Magnetic Characteristics, Angew. Chem. Int. Ed, 41, 2, 281.

11 M.R. Maurya et al, 2008, Immobilisation of oxovanadium (IV), dioxomolybdenum (VI) and copper (II) complexes on polymers for the oxidation of styrene, cyclohexene and ethylbenzene, App.Cat.A-General, 351, 2, 239.

12 M. J. Haanepen et al, 1997, VAPO as catalyst for liquid phase oxidation reactions. Part1: preparation, characterisation and catalytic performance, App.Cat.A-General, 152, 183. 